Check for updates

Cite this: RSC Adv., 2019, 9, 9486

\title{
Potent $\alpha$-amylase inhibitory activity of sprouted quinoa-based yoghurt beverages fermented with selected anti-diabetic strains of lactic acid bacteria
}

\author{
Obaroakpo Joy Ujiroghene, ${ }^{\text {ab }}$ Lu Liu, ${ }^{c}$ Shuwen Zhang, ${ }^{a}$ Jing Lu, ${ }^{a}$ Cai Zhang, \\ Xiaoyang Pang (iD ${ }^{* a}$ and Jiaping $L v^{* a}$
}

\begin{abstract}
The in vitro inhibitory effect of sprouted quinoa yoghurt beverages (QYB) fermented with anti-diabetic lactic acid bacteria on $\alpha$-amylase was investigated. In vitro studies using porcine pancreatic $\alpha$-amylase showed that quinoa yoghurt beverages fermented with Lactobacillus casei Zhang and Lactobacillus casei SY13 dose-dependently inhibited the activities of $\alpha$-amylase. The saponin content, reducing and total sugars were also quantified to determine their potency as anti-hyperglycemic agents against type 2 diabetes mellitus. The saponin contents of the yoghurt beverages were relatively low at a range of $0.19-0.41 \%$, and significantly reduced as germination time increased. Germination significantly decreased the reducing sugars in all samples. A total of 4 sugars were identified using HPLC. Quinoa yoghurt beverages can be targeted as a potential dual-inhibitory strategy to attenuate type 2 diabetes mellitus by their ability to inhibit $\alpha$-amylase activity as well as reduce or prevent hyperglycemic conditions associated with elevated levels of sugar glucose in the blood.
\end{abstract}

Received 7th December 2018

Accepted 2nd March 2019

DOI: $10.1039 / c 8 r a 10063 b$

rsc.li/rsc-advances have been found to be effective in managing postprandial hypoglycemia.

Quinoa has raised the interests of researchers with respect to better blood sugar regulation, as well as several other aspects of blood sugar metabolism. Quinoa have also been evaluated for their potential in lowering the risk of T2DM by inhibiting hyperglycemic and hypertensive activities in vitro. ${ }^{2}$ This is expected, since it is a good source of protein and fiber, and low glycemic index (GI).

Kaur \& Tanwar $^{3}$ reported a low inhibitory potential of quinoa beverages against $\alpha$-amylase enzyme. According to his report, even though quinoa beverages had potential health benefits, germinated quinoa beverages seemed more effective in the management of T2DM and hypertension. Many studies have reported amylase activity in cereal grains during germination. Simple processing methods such as fermentation and germination has shown to influence $\alpha$-amylase inhibitory activity in cereals. ${ }^{4}$

Recently, probiotics such as lactic acid bacteria (LAB) having anti-diabetic potentials are used in inoculating natural products to further ameliorate their anti-diabetic activities. Probiotic fermented products can be useful as a complementary or adjuvant therapy in the treatment of diabetics. Due to the nutritional and health benefits of quinoa, they have currently been included in several health promoting probiotic products such as yoghurt. This present study therefore intended to explore the potential of germinated quinoa-based yoghurt beverage, influenced by inoculation with selected anti-diabetic strains of LAB, as prospective inhibitor of $\alpha$-amylase with antihyperglycemic mechanism of action. 


\section{Materials and method}

\section{Materials}

$1000 \mathrm{~g}$ each of two varieties of quinoa (Mengli 1 and Mengli 2 cultivars) were purchased from Inner Mongolia Province, China. The cultivars were selected based on their low saponin content. All reagents and chemicals used in this study are of analytical grade.

\section{Inoculant strains and culture condition}

Lactobacillus bulgaricus ljj, Lactobacillus casei SY13 used for this investigation are previously established anti-diabetic strains isolated from cow milk yoghurt in Dairy Research Laboratory of Chinese Academy of Agricultural Sciences, Beijing, China. Lactobacillus casei Zhang was donated by Professor Zhang Heping from Inner Mongolia Agricultural University. The LAB were singly resuscitated and propagated by inoculating $0.1 \mathrm{ml}$ in $100 \mathrm{ml}$ sterile MRS broth (AoBoxing, Beijing, China), and incubated at $30{ }^{\circ} \mathrm{C}$ for $24 \mathrm{~h}$. Each LAB was sub-cultured twice, the cells were separated from the broth by centrifuging $(7500 \times$ $g$ for $10 \mathrm{~min}$ at $4{ }^{\circ} \mathrm{C}$ ), and then washed with sterile quarter strength Ringer's solution. The cells were re-harvested by centrifugation $\left(12000 \times g\right.$ for $15 \mathrm{~min}$ at $\left.4{ }^{\circ} \mathrm{C}\right)$, and re-suspended in $5 \mathrm{ml}$ of distilled water. Finally, the number of LAB cells in each suspension was adjusted to a range of $6 \log \mathrm{CFU} \mathrm{ml} \mathrm{m}^{-1}(\lambda=$ $600 \mathrm{~nm}, \mathrm{OD}=0.8$ for Lactobacillus casei Zhang and Lactobacillus bulgaricus ljj, and OD $=1$ for Lactobacillus casei SY13) using spectrophotometer (Tecan Spark 20M).

\section{Quinoa sprouted milk}

Cleaned and sorted quinoa grains of Mengli 1 cultivar (M1C) and Mengli 2 cultivar (M2C), were separately pre-conditioned by soaking for $2 \mathrm{~h}(1: 2)$ in distilled water, and then washed till it became foamless to remove saponin and ease the germination process. The grains were rinsed, spread on germination chambers at ambient temperature and left to germinate at varying time intervals ( $0 \mathrm{~h}, 24 \mathrm{~h}$ and $72 \mathrm{~h}$ ). The germinated quinoa seeds were blended at 25\% w/v using attrition mill (YL7112 model), and the milk was extracted from the pulp using a nut milk bag.

\section{Quinoa yoghurt beverage production}

The extracted milk from germinated quinoa was separately inoculated with selected strains of LAB (Lactobacillus bulgaricus ljj, Lactobacillus casei Zhang, Lactobacillus casei SY13) for the production of quinoa yoghurt beverage (QYB). At germination time of $0 \mathrm{~h}, 24 \mathrm{~h}$ and $72 \mathrm{~h}$, quinoa milk was extracted and used in the production of yoghurt beverages. The LAB were inoculated at a concentration of $6 \log \mathrm{CFU} \mathrm{ml} \mathrm{m}^{-1}$, and fermented for $8 \mathrm{~h}$ at $42{ }^{\circ} \mathrm{C}$. A combined starter culture of Lactobacillus delbrueckii bulgaricus and Streptococcus thermophilus was used as control.

\section{Methods}

\section{Physico-chemical analyses}

PH was determined using Mettler Toledo $\mathrm{pH}$ meter (Delta 320). The total soluble solids were determined using a refractometer (VBR-BRIS series). The titratable acidity (TTA) was expressed as the volume (in $\mathrm{ml}$ ) of $\mathrm{NaOH}$ solution used in titration, to the nearest $0.05 \mathrm{ml}$.

\section{Preparation of quinoa yoghurt extract}

Extract from QYB was prepared by diluting $1 \mathrm{ml}$ of sample in $4 \mathrm{ml}$ of Tris- $\mathrm{HCl}$ (pH 8.7), held for $1 \mathrm{~h}$ at $4{ }^{\circ} \mathrm{C}$ while vortexing at intervals of $15 \mathrm{~min}$. The mixture was then centrifuged at 20000 $\times g$ for $20 \mathrm{~min}$. The resulting supernatant was filtered through a $0.22 \mu \mathrm{m}$ pore size filter, and stored at $-4{ }^{\circ} \mathrm{C}$ until further analysis.

\section{Estimation of total saponins}

Total saponin in QYB was determined by the slightly modified method of Dou. ${ }^{5} 500 \mu$ of $0.5 \%$ anisaldehyde reagent was added to $500 \mu \mathrm{l}$ of sample extract, and allowed to stand for $10 \mathrm{~min}$. $2 \mathrm{ml}$ of $50 \%$ sulphuric acid was the added, and the tubes thoroughly mixed. The tubes were placed in a water bath at a constant temperature of $60{ }^{\circ} \mathrm{C}$ for $10 \mathrm{~min}$, and then cooled. Absorbance was taken at $435 \mathrm{~nm}$. Saponin content was calculated as saponin equivalent from the calibration curve of standard saponin of known concentrations.

\section{Extraction of sugars from yoghurt}

Quinoa yoghurt samples were defatted by centrifugation at 6500 $\times g$ for $15 \mathrm{~min}$ at $5{ }^{\circ} \mathrm{C}$, and then kept in an ice bath for $30 \mathrm{~min}$. The lipid layer supernatant was removed using $0.22 \mu \mathrm{m}$ filter syringe. The removal of protein fraction was carried out by the addition of two volumes of cold ethanol to the skimmed yoghurt samples. The solutions were then re-centrifuged for $30 \mathrm{~min}$ at $5{ }^{\circ} \mathrm{C}$. Supernatants were collected, and the ethanol was removed in a rotary evaporator at $37^{\circ} \mathrm{C}$. The carbohydrate fraction of sugars in the aqueous solution was stored at $-18^{\circ} \mathrm{C}$ for further analysis.

\section{Determination of reducing sugar by DNS method}

The reducing sugars in QYB were determined using 3,5-dinitrosalicylic acid (DNS) calorimetric method. $1 \mathrm{ml}$ of sample extract was measured into a test tube, into which $1 \mathrm{ml}$ of DNS agent was added, and then kept in a boiling water bath for 5 min. The resulting mixture was allowed to cool to room temperature $\left(25^{\circ} \mathrm{C}\right)$ in a cold water bath, and $10 \mathrm{ml}$ of distilled water was added. Absorbance was measured at $540 \mathrm{~nm}$. The values obtained were interpolated with standard of known glucose concentrations.

\section{Determination of total sugar by phenol-sulphuric acid method}

The total sugars in QYB were determined using the phenolsulphuric acid method. $50 \mu$ l of sample extract was put in a test tube and then made up to $500 \mu \mathrm{l} .0 .3 \mathrm{ml}$ of $5 \%$ phenol solution and $1.8 \mathrm{ml}$ of concentrated sulphuric were added through the tube wall. The resultant solution was cooled to room temperature for $15 \mathrm{~min}$, until the colour change occurred. Sugar content was determined by referring to the glucose standard solution (3.125-50 $\left.\mu \mathrm{g} \quad \mathrm{ml}^{-1}\right)$. Absorbance was measured at $490 \mathrm{~nm}$ with a spectrophotometer. 


\section{Separation and quantification of sugars by HPLC-RI}

The sugars in QYB were identified and quantified by HPLC with refractometric detection (Agilent 1260). The analytical column used was $\mathrm{NH}_{2}$ with mobile phase $(4.6 \times 250 \mathrm{~mm})$ with a precolumn. The mobile phase was acetonitrile and re-distilled

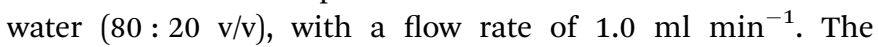
column temperature was $35^{\circ} \mathrm{C}$, and injection volume was $20 \mu \mathrm{l}$. The chromatographic peaks were identified by using the retention time of the reference standards.

\section{$\alpha$-Amylase inhibition assay}

The inhibition of $\alpha$-amylase activity was determined using the modified method of Nickavar \& Yousefian. ${ }^{6}$ The enzyme solution used for this assay was prepared using 0.5 unit per ml of $\alpha$ amylase in purified water. Starch solution of $0.1 \% \mathrm{w} / \mathrm{v}$ was prepared by stirring $0.1 \mathrm{~g}$ of potato starch in $100 \mathrm{ml} 16 \mathrm{mM}$ sodium acetate buffer. The calorimetric reagent was prepared by the mixture of $5.3 \mathrm{M}$ sodium potassium tartrate solution (in $2 \mathrm{M} \mathrm{NaOH}$ ) and $96 \mathrm{mM} \mathrm{3,5-dinitro} \mathrm{salicylic} \mathrm{acid} \mathrm{solution.}$ Appropriately diluted sample solutions at various concentrations (100-400 $\mu \mathrm{l} \mathrm{ml}^{-1}$ ) were added to $1 \mathrm{ml}$ of starch solution, and then allowed to stand for $10 \mathrm{~min}$. The reaction was furthered by the addition of $500 \mu \mathrm{l}$ of enzyme solution, and allowed to stand for another 10 min under alkaline conditions at $25{ }^{\circ} \mathrm{C}$. The reaction was then terminated by the addition of $1 \mathrm{ml}$ of colorimetric reagent, and then incubated in boiling water for $5 \mathrm{~min}$. The reaction mixture was allowed to cool to room temperature, and then diluted by adding $10 \mathrm{ml}$ of distilled water. Alpha amylase inhibitory activity was measured using spectrophotometer at $540 \mathrm{~nm}$. 100\% enzyme activity was used as control, and conducted in a similar way by replacing extract with DMSO. Standard drug acarbose, was used as positive control. The absorbance $(A)$ due to maltose generated was calculated as $A_{540 \mathrm{~nm}}$ control or sample $=A_{540 \mathrm{~nm}}$ test $-A_{540 \mathrm{~nm}}$ blank.

$$
\text { Inhibition was calculated as : }\left(1-\frac{B}{A}\right) \times 100 \%
$$

where $A=$ absorbance of control (with the exclusion of test samples). $B=$ absorbance of reaction mixture (with the inclusion of test samples).

\section{Results and discussion}

Diabetes mellitus is generally characterized by high concentrations of blood sugar levels, which can cause health complications such as organ failures, as well as several cardiovascular diseases. Therefore, inhibition of carbohydrate-hydrolyzing enzymes such as $\alpha$-amylase will decrease postprandial hyperglycemia by retarding the absorption of glucose.

\section{Effect of inoculant strain on $\mathrm{pH}$, titratable acidity and total solids of QYB}

The results of the physico-chemical properties of QYB are represented in Table 1 . The inoculant strains used in the fermentation of quinoa yoghurt beverage (QYB) significantly $(p<0.05)$ influenced the $\mathrm{pH}$ and titratable acidity (TA). Generally, suitable probiotics are characterized by their ability to tolerate low $\mathrm{pH} .^{7,8}$ As expected, the $\mathrm{pH}$ of QLB, QLCZ and QLCSY13 were lower than those of QSC. In M1C, the $\mathrm{pH}$ of all QYB increased with germination time at ranges of 3.90 $\pm 0.01-4.17 \pm 0.01$ (QSC), $3.37 \pm 0.01-3.59 \pm 0.01$ (QLB), $3.47 \pm 0.01-3.74 \pm 0.01$ (QLCZ), and $3.17 \pm 0.01-3.29 \pm 0.01$ (QLCSY13). In M2C, pH increased in QYB produced at $24 \mathrm{~h}$ when compared to QYB at $0 \mathrm{~h}$. However, a slight decrease was observed at $72 \mathrm{~h}$.

A negative correlation (data not shown) was recorded between $\mathrm{pH}$ and TA of all QYB in such a way that as $\mathrm{pH}$ reduced, TA increased. This could be attributed to the post acidification processes associated with lactic acid formation and the growth of LAB during fermentation. The variations in $\mathrm{pH}$ within QYB is inoculant strain-dependent. ${ }^{7}$

The combined starter culture used in this study (Lactobacillus delbrueckii bulgaricus and Streptococcus thermophilus) is the typical starter culture used in yoghurt production, ${ }^{9}$ hence the highest total soluble solids (TSS) recorded in QSC of both M1C and M2C is expected. However compared with QSC (15.67 $\pm 0.01 \%$ ), at a mean value of $13.33 \pm 0.01 \%$ QLCZ recorded highest TSS; while in M2C, TSS was highest in QLCSY13 at a mean value of $11.70 \pm 0.01 \%$ when compared with QSC (12.30 $\pm 0.01 \%$ ). The total soluble solids (TSS) in all QYB decreased with increase in germination time.

\section{Quantification of reducing sugars in QYB}

Reducing sugars significantly $(p<0.05)$ increased in all QYB when compared to RQM. In M1C, reducing sugars increased from $1.11 \%$ (RQM) to $2.42 \%$ (QSC), 2.76\% (QLB), 2.26\% (QLCZ), and $2.95 \%$ (QLCSY13); while in M2C increased from $0.79 \%$ (RQM) to $2.30 \%$ (QSC), 1.56\% (QLB), 1.44\% (QLCZ) and 1.53\% (QLCSY13). Germination significantly $(p<0.05)$ decreased the reducing sugars in all QYB. From our results, it was observed that the anti-diabetic strains inoculated-QYB having lowest reducing sugars, had a corresponding highest TA and lowest $\mathrm{pH}$. This is expected as LAB strains utilized most of the sugars during fermentation, thus releasing more lactic acids and lowering of $\mathrm{pH}$. This result is in agreement with those of Song, Shin \& Baik. ${ }^{10}$ Apart from the influence of germination on the ability of sugar reduction by LAB, strain-dependence may also be a factor. Reducing sugars are the most important substrate for microorganisms to undergo fermentation.

There were significant differences $(p<0.05)$ in the sugar profile of all QYB, which could be as a result of variations in inoculant strains (Table 2). Changes in germination time also influenced the sugar composition (most especially glucose), and caused significant reductions as germination time increased. Glucose and fructose (reducing sugars) are the major sugars in all QYB. The richness of QYB in reducing sugars suggests the existence of high invertase activity, which considerably reduced its sucrose content.

\section{HPLC-RI detection of total sugars}

A reliable HPLC method with refractive index detection was used in the identification of sugars in all QYB. Galactose, 


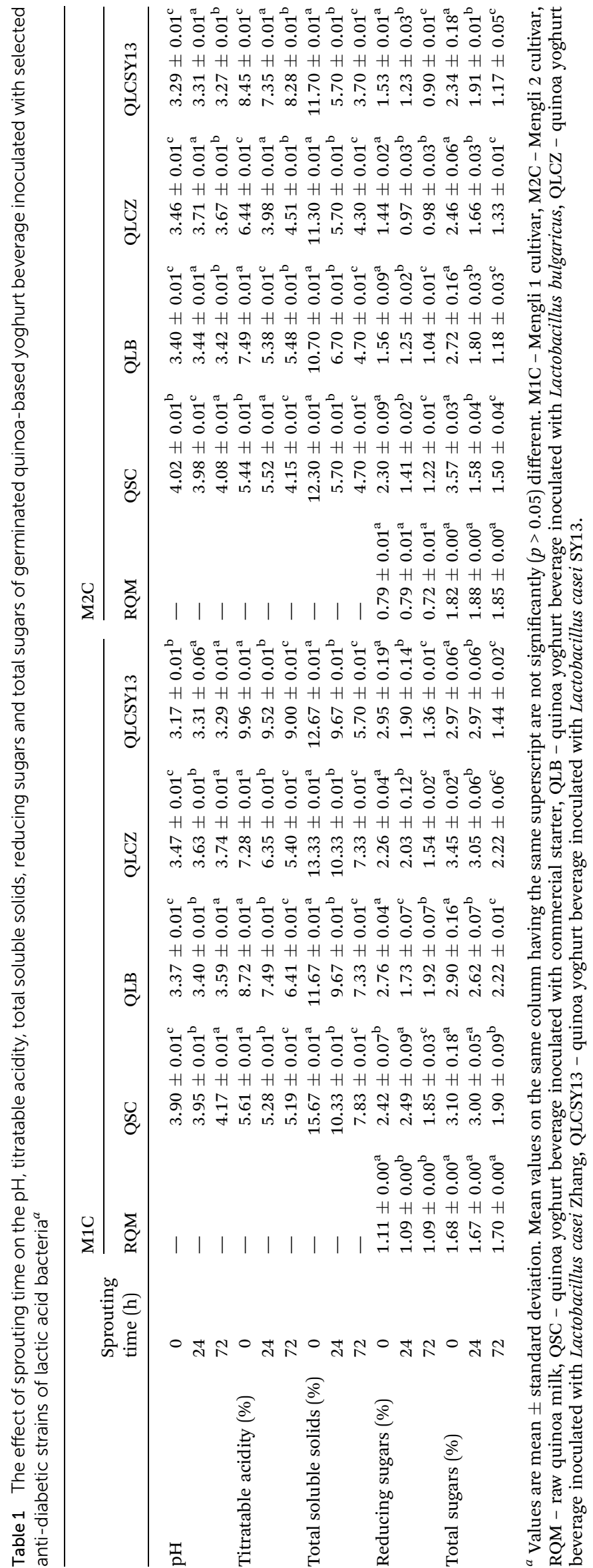


Table 2 Quantity of sugars in germinated quinoa-based yoghurt beverages determined by high performance liquid chromatography with refractive index detection $(\mathrm{HPLC}-\mathrm{RI})^{a}$

SUGAR CONTENT $\left(\mu \mathrm{g} \mathrm{ml}^{-1}\right)$

\begin{tabular}{|c|c|c|c|c|c|c|c|c|c|}
\hline \multirow[b]{2}{*}{ Sugar } & \multirow{2}{*}{$\begin{array}{l}\text { Sprouting } \\
\text { time }(\mathrm{h})\end{array}$} & \multicolumn{4}{|l|}{ M1C } & \multicolumn{4}{|l|}{ M2C } \\
\hline & & QSC & QLB & QLCZ & QLCSY13 & QSC & QLB & QLCZ & QLCSY13 \\
\hline \multirow[t]{3}{*}{ Galactose } & 0 & 0.04 & ND & 0.01 & 0.01 & 0.06 & 0.01 & 0.01 & ND \\
\hline & 24 & 0.05 & ND & 0.01 & ND & 0.06 & 0.01 & 0.01 & ND \\
\hline & 72 & 0.05 & 0.01 & ND & 0.01 & 0.05 & 0.01 & ND & 0.01 \\
\hline \multirow[t]{3}{*}{ Glucose } & 0 & 10.84 & 7.24 & 9.34 & 10.48 & 9.52 & 9.30 & 8.99 & 7.59 \\
\hline & 24 & 11.80 & 8.26 & 10.99 & 6.69 & 5.26 & 5.72 & 6.51 & 6.23 \\
\hline & 72 & 7.85 & 5.86 & 8.90 & 6.65 & 3.75 & 4.03 & 2.40 & 1.97 \\
\hline \multirow[t]{3}{*}{ Sucrose } & 0 & 0.41 & 0.34 & 0.96 & 1.08 & 0.02 & 0.47 & 0.75 & 0.26 \\
\hline & 24 & 0.63 & 0.41 & 0.72 & 0.21 & 0.05 & 0.11 & 0.25 & 0.10 \\
\hline & 72 & 0.41 & 0.30 & 0.51 & 0.56 & 0.19 & 0.29 & 0.26 & 0.17 \\
\hline \multirow[t]{3}{*}{ Fructose } & 0 & 0.10 & ND & 0.04 & 0.08 & 0.09 & 0.05 & 0.03 & 0.06 \\
\hline & 24 & 0.33 & ND & ND & ND & 0.24 & 0.04 & 0.02 & ND \\
\hline & 72 & 0.33 & 0.10 & 0.14 & 0.03 & 0.26 & 0.04 & 0.02 & 0.03 \\
\hline \multirow{3}{*}{$\begin{array}{l}\text { Total sugar } \\
\text { content }\end{array}$} & 0 & 11.39 & 7.58 & 10.35 & 11.65 & 9.69 & 9.83 & 9.78 & 7.91 \\
\hline & 24 & 12.81 & 8.67 & 11.72 & 6.90 & 5.61 & 5.88 & 6.79 & 6.33 \\
\hline & 72 & 8.64 & 6.27 & 9.55 & 7.25 & 4.25 & 4.37 & 2.68 & 2.18 \\
\hline
\end{tabular}

${ }^{a}$ Values are mean of triplicate determinations. ND-not detected.
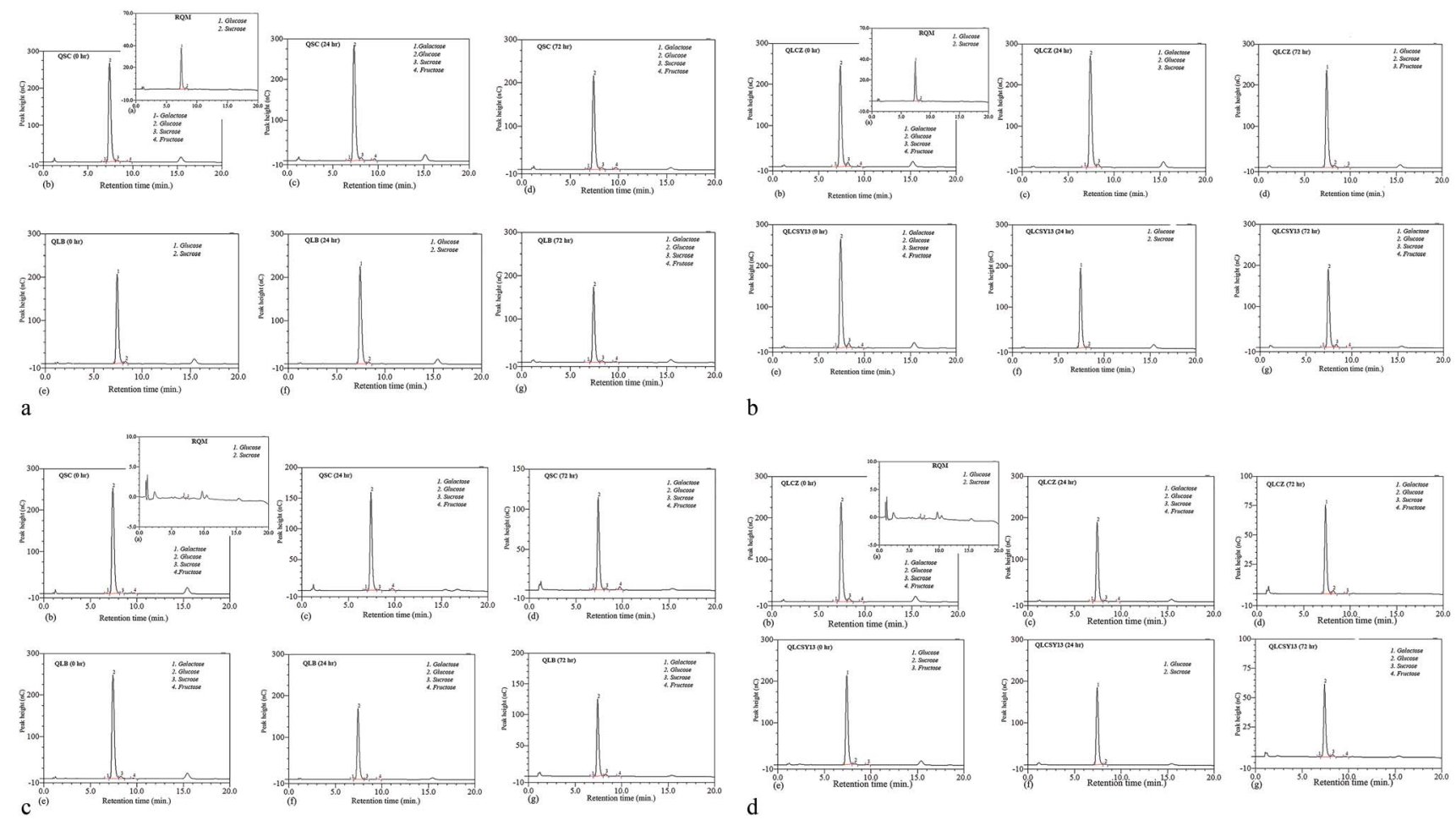

b
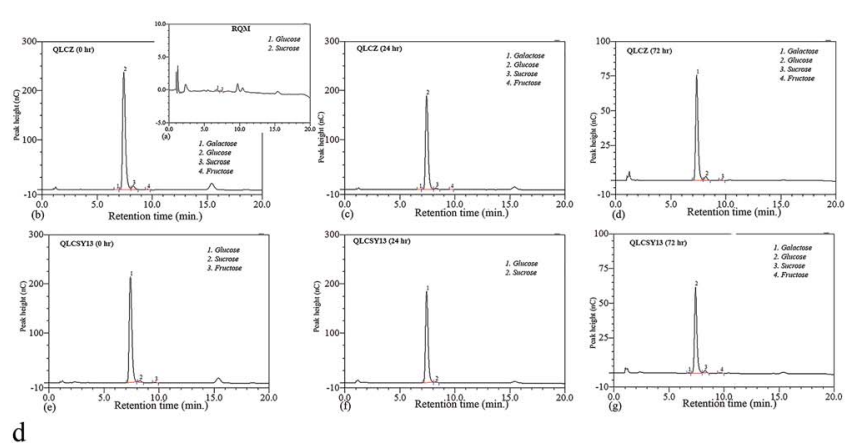

Fig. 1 (a) HPLC-RI chromatogram of sugars identified in quinoa yoghurt beverages (QYB) of Mengli 1 cultivar (M1C) at $0 \mathrm{~h}, 24 \mathrm{~h}$ and $72 \mathrm{~h}$. The conditions of the experiment are stated in the main text. RQM represents raw quinoa milk, QSC represents QYB inoculated with commercial starter culture, and QLB represents QYB inoculated with Lactobacillus bulgaricus ljj. (b) HPLC-RI chromatogram of sugars identified in quinoa yoghurt beverages (QYB) of Mengli 1 cultivar (M1C) at $0 \mathrm{~h}, 24 \mathrm{~h}$ and $72 \mathrm{~h}$. The conditions of the experiment are stated in the main text. RQM represents raw quinoa milk, QLCZ represents QYB inoculated with Lactobacillus casei Zhang, and QLCSY13 represents QYB inoculated with Lactobacillus casei SY13. (c) HPLC-RI chromatogram of sugars identified in quinoa yoghurt beverages (QYB) of Mengli 2 cultivar (M2C) at $0 \mathrm{~h}, 24 \mathrm{~h}$ and $72 \mathrm{~h}$. The conditions of the experiment are stated in the main text. RQM represents raw quinoa milk, QSC represents QYB inoculated with commercial starter culture, and QLB represents QYB inoculated with Lactobacillus bulgaricus lj. (d) HPLC-RI chromatogram of sugars identified in quinoa yoghurt beverages (QYB) of Mengli 2 cultivar (M2C) at $0 \mathrm{~h}, 24 \mathrm{~h}$ and $72 \mathrm{~h}$. The conditions of the experiment are stated in the main text. RQM represents raw quinoa milk, QLCZ represents QYB inoculated with Lactobacillus casei Zhang, and QLCSY13 represents QYB inoculated with Lactobacillus casei SY13. 
glucose, sucrose and fructose were detected at retention time of $6.79 \mathrm{~min}, 7.41 \mathrm{~min}, 8.27 \mathrm{~min}$, and $9.66 \mathrm{~min}$ respectively. Fig. 1(a-d) showed the HPLC chromatogram of sugars in all QYB samples. Cereals usually contain only small amounts of sugars prior to germination, since most sugars transported to the seeds are normally converted to starch. ${ }^{11}$ Complex sugars and starch are broken down during germination as a result of saccharification, resulting in an increase in the amount of glucose. Also, increase in glucose might be as a result of the breakdown of sucrose. In RQM, only glucose and sucrose were detected in both M1C and M2C. At $0 \mathrm{~h}$, fructose and galactose were then released in some QYB at minimal concentrations, while glucose increased. At $24 \mathrm{~h}$ of germination, QYB recorded high glucose peak which later reduced with increased germination time $(72 \mathrm{~h})$.

Even though the glucose content was generally higher than other sugars in QYB, the ability for the anti-diabetic strains to reduce its content was observed in our results due to its reduction when compared with QSC. In M1C, the glucose content reduced in QLB (7.24\%), QLCZ (9.34\%), and QLCSY13 (10.48\%), when compared with QSC (10.84\%). Also in M2C, a decline in glucose content was also recorded in QLB (9.30\%), QLCZ (8.99\%) and QLCSY13 (7.59\%), when compared with QSC (9.52\%). While QLB had lowest glucose in M1C, QLCSY13 had lowest glucose in M2C. Several studies have indicated that probiotics can lower glucose and insulin levels in those with diabetics. ${ }^{12}$

\section{Influence of germination on saponin content in QYB}

Saponins of food cereals usually undergo certain changes during washing, germination and cooking. ${ }^{13}$ Fig. 2 represents the saponin contents of QYB, as well as the influence of germination on its composition. Generally in quinoa seeds, saponin ranges from 0.1 to $5 \%{ }^{14}$ Based on this report, it could be observed from our results that the saponin content in QYB was relatively low, at ranges of $0.19-0.41 \%$. Valencia-Chamorro ${ }^{15}$ reported that saponin of quinoa seeds are usually reduced to minimal levels that are apparently non-toxic to humans after washing. Our studies also showed slight differences in saponin content at $0 \mathrm{~h}$ between all
QYB in M1C when compared to RQM $(0.33 \pm 0.00)$, at mean values of $0.35 \pm 0.00 \%$ (QSC), $0.33 \pm 0.01 \%$ (QLB), $0.31 \pm 0.00 \%$ (QLCZ), and $0.31 \pm 0.01 \%$ (QLCSY13). Similarly, in comparison to RQM $(0.41 \pm 0.00 \%)$ of M2C, all QYB had significant differences $(p<$ $0.05)$ at mean values of $0.39 \pm 0.06 \%, 0.31 \pm 0.00 \%, 0.33 \pm 0.00 \%$, and $0.34 \pm 0.01 \%$ in QSC, QLB, QLCZ, and QLCSY13 respectively.

Germination, a valid tool for decreasing bitterness in quinoa due to the formation of sugar, ${ }^{16}$ and was also evident in our study due to the decreased saponin content recorded at increased germination time. According to our results however, highest reduction of saponin in M1C was reported in QLCSY13. Likewise, QLCSY13 also had lowest saponin content in M2C and was not significantly different $(p>0.05)$ from QLCZ. Even though saponin is sometimes characterized by a slightly bitter flavor, its potential benefit in reducing the risk of cardiovascular diseases has severally been reported. Dietary saponins are generally regarded as safe, and are therefore not a concern for human health. ${ }^{17}$

\section{Inhibitory action of QYB against $\alpha$-amylase}

The $\alpha$-amylase inhibition activities of QYB of M1C and M2C are represented in Fig. 3. Our study showed a dose-dependent inhibitory activity of all QYB against $\alpha$-amylase. As concentration increased inhibitory actions of QYB against $\alpha$-amylase activity also increased. Even though its inhibitory level at $100 \mu \mathrm{l}$ was lower than the positive control (acarbose) used in this study, at concentration of $400 \mu \mathrm{l}$, its inhibitory level became remarkably higher. During germination, endogenous $\alpha$-amylase is inhibited as a result of starch granule synthesis. Thus, our results showed highest inhibition activity at $0 \mathrm{~h}$ in agreement with the reports of Mulimani. ${ }^{4}$ However, it is important to accentuate the QYB with the significantly average $\alpha$-amylase inhibitory activities. This is potentially important to reduce to minimum, the side effects of excessively high $\alpha$-amylase inhibition usually encountered in the use of synthetic drugs. ${ }^{18}$

\section{The half maximal inhibitory concentration $\left(\mathrm{IC}_{50}\right)$ of $\mathrm{QYB}$ against $\alpha$-amylase}

Anti-diabetic strains of LAB used in this study, decreased the $\mathrm{IC}_{50}$ values (Table 3). It has been postulated that the lower the $\mathrm{IC}_{50}$
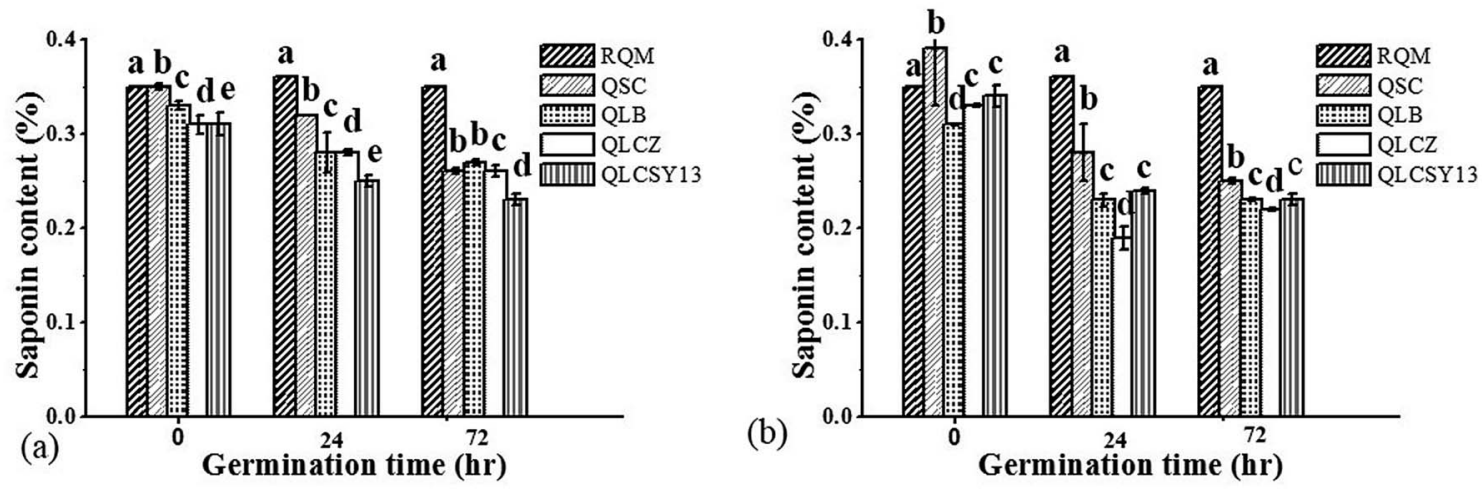

Fig. 2 (a) Saponin content (\%) in quinoa yoghurt beverages (QYB) of Mengli 1 cultivar. (b) Saponin content (\%) in quinoa yoghurt beverages (QYB) of Mengli 2 cultivar. RQM - raw quinoa milk, QSC - quinoa yoghurt beverage inoculated with commercial starter, QLB - quinoa yoghurt beverage inoculated with Lactobacillus bulgaricus ljj, QLCZ - quinoa yoghurt beverage inoculated with Lactobacillus casei Zhang, QLCSY13 quinoa yoghurt beverage inoculated with Lactobacillus casei SY13. 

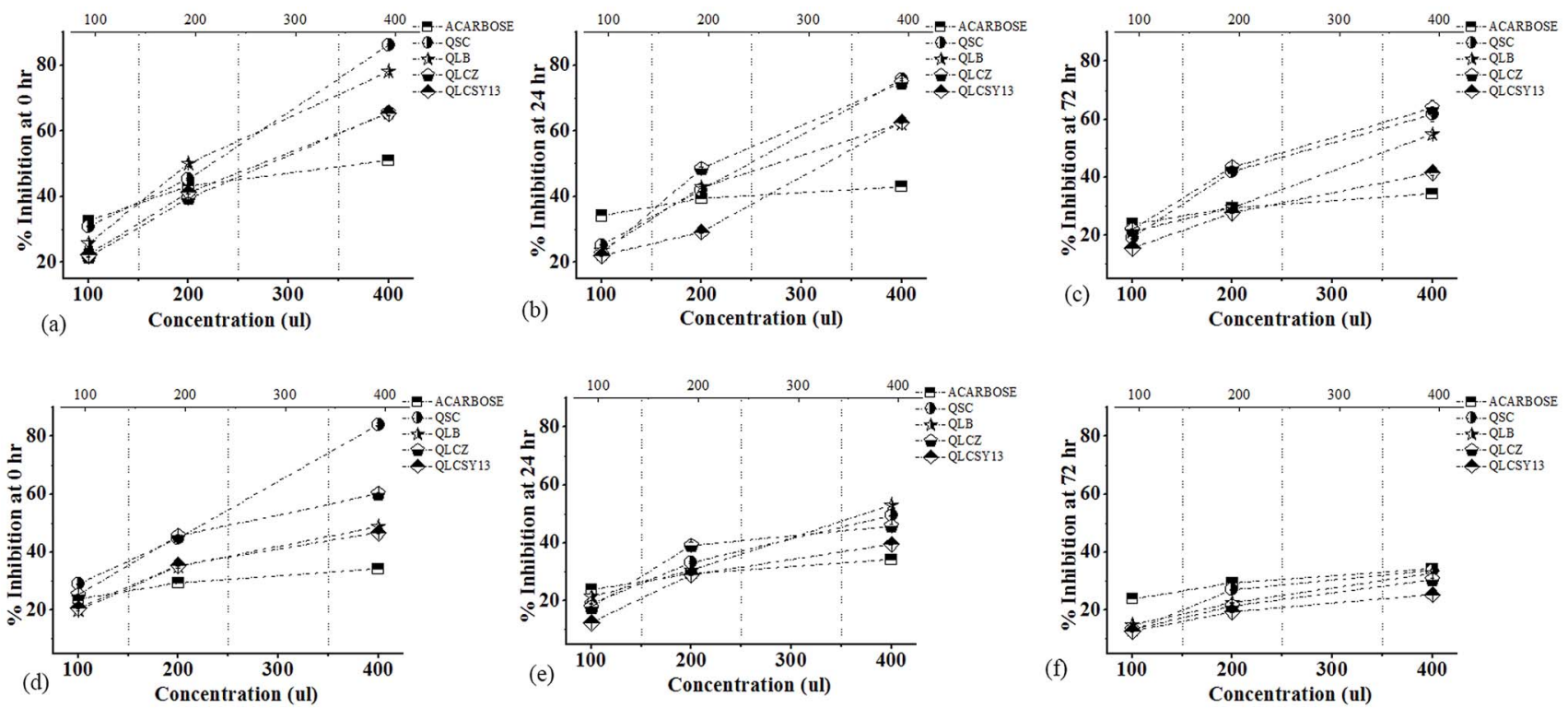

Fig. 3 Alpha-amylase inhibitory activity of quinoa yoghurt beverages (QYB) in M1C and M2C. The values presented are expressed as mean \pm standard deviation of triplicate experiments. QSC - quinoa yoghurt beverage inoculated with commercial starter, QLB - quinoa yoghurt beverage inoculated with Lactobacillus bulgaricus ljj, QLCZ - quinoa yoghurt beverage inoculated with Lactobacillus casei Zhang, QLCSY13 quinoa yoghurt beverage inoculated with Lactobacillus casei SY13. Acarbose represents positive control.

Table $3 \alpha$-Amylase inhibitory activities and $I_{50}$ values of quinoa-based yoghurt beverage in relation to sprouting time ${ }^{a}$

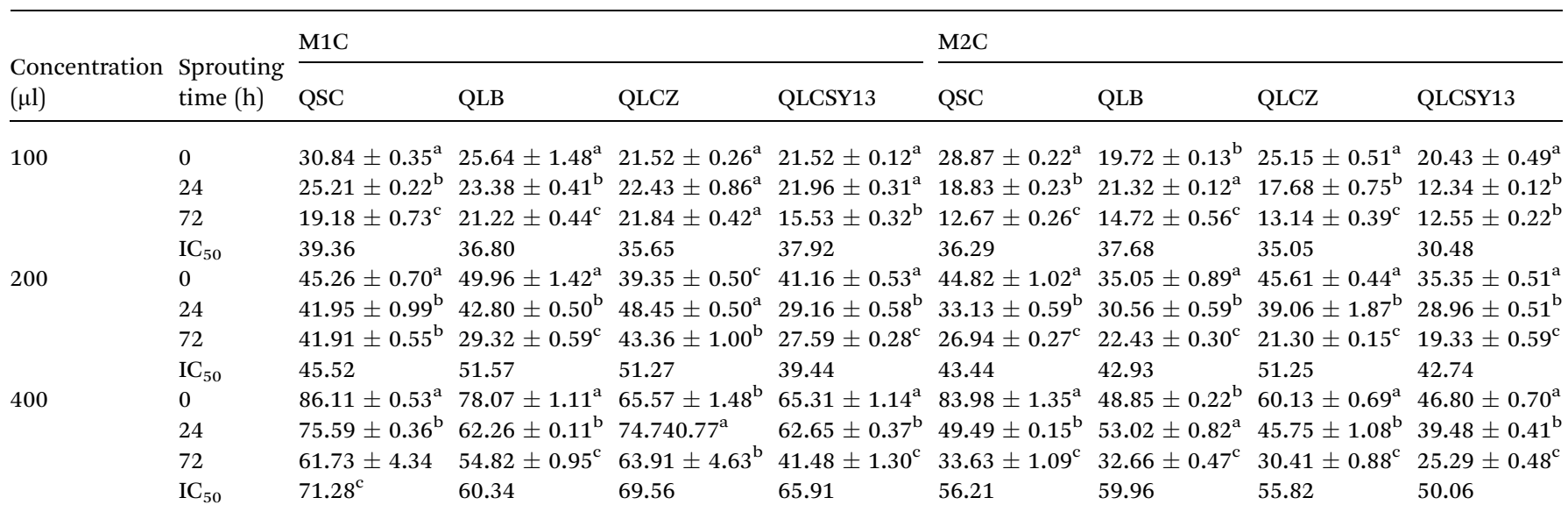

${ }^{a}$ Values are mean \pm standard deviation. Mean values on the same column having the same superscript are not significantly $(p>0.05)$ different.

values, the stronger the inhibitor. ${ }^{19}$ In $\mathrm{M} 1 \mathrm{C}$ and M2C, highest inhibition activity was recorded in $\mathrm{QLCZ}\left(\mathrm{IC}_{50}=35.65 \mathrm{mg} \mathrm{ml}^{-1}\right)$ and QLCSY13 $\left(\mathrm{IC}_{50}=30.48 \mathrm{mg} \mathrm{ml}^{-1}\right.$ ) respectively. At $\mathrm{IC}_{50}$ value of $39.36 \mathrm{mg} \mathrm{ml}{ }^{-1}$, QSC recorded lowest $\alpha$-amylase inhibition activity. However, lowest activity in M2C was recorded in QLB $\left(\mathrm{IC}_{50}=37.68 \mathrm{mg} \mathrm{ml}^{-1}\right)$, and was not significantly different $(p>$ $0.05)$ from the activity in $\mathrm{QSC}\left(\mathrm{IC}_{50}=36.29 \mathrm{mg} \mathrm{ml}^{-1}\right)$.

\section{Correlation between saponin content and $\alpha$-amylase inhibition activity}

The inhibitory effect of QYB may be linked to the presence of saponins, which may be acting individually or in synergy. A strong positive correlation (results not included) was obtained between saponin content and $\alpha$-amylase inhibition in QYB. Ishnava and Motisariya ${ }^{20}$ reported that the presence of phytochemicals in plant extracts might be responsible for the inhibition of $\alpha$ amylase activity. The results of our study showed that increase in saponin content of QYB resulted in corresponding increase in $\alpha$ amylase inhibition. Our findings agrees with the reports of Kazeem, Ogunbiyi \& Ashafa, ${ }^{21}$ that phytochemicals such as saponins and terpenoids from plants are strong inhibitors of $\alpha$ glucosidase and $\alpha$-amylase. Anti-hyperglycemic activity of saponin has been attributed to its ability to induce the release of insulin from the pancreas; thereby partially regulating the use and storage of glucose and fat in the body. ${ }^{22}$ However, further studies are needed to validate the specific constituent of saponin in QYB responsible for reducing hypoglycemia. 


\section{Conclusion}

In this study, our results showed that QYB exhibited strong inhibition activity in a dose-dependent manner, and is thus inferred to be an effective $\alpha$-amylase inhibitor. Its inhibitory activity against $\alpha$-amylase was comparable to positive control (acarbose). The presence of saponin; a phytochemical component present in quinoa, apparently influenced the inhibitory activity of QYB against $\alpha$-amylase. Yoghurt as a functionally bioactive food capable of inhibiting $\alpha$-amylase, as well as reducing glucose metabolism may be used to attenuate health complications in T2DM.

Although all anti-diabetic LAB strains tested in this study attenuated anti-diabetic effect through $\alpha$-amylase inhibition and hyperglycemic reduction in vitro, QLCZ and QLCSY13 were reported to possess highest potential against anti-diabetic effect in T2DM. The conclusion was based on their notably highest $\alpha$ amylase inhibition activity, as well as their ability to reduce total sugars in QYB. These results suggest that the anti-diabetic effect of LAB on $\alpha$-amylase is dependent on lactic acid bacteria strain.

Conclusively, QYB can be targeted as a potential dualinhibitory strategy to attenuate T2DM by their ability to inhibit $\alpha$-amylase activity as well as reduce or prevent hyperglycemic conditions associated with elevated levels of sugar glucose in the blood.

\section{Conflicts of interest}

The authors confirm that this article content has no conflicts of interest.

\section{Funding}

This work was financially supported by National Key R\&D Program of China (2017YFC1600903) and National Natural Science Foundation of China (31871833).

\section{References}

1 M. A. Alsuhaibani, Middle East Afr. J. Ophthalmol., 2016, 6(2), 341-348.
2 Y. Tang, X. Li, P. X. Chen, B. Zhang, M. Hernandez, H. Zhang and R. Tsao, Food Chem., 2014, 1-32.

3 I. Kaur and B. Tanwar, Rom. J. Diabetes Nutr. Metab. Dis., 2016, 23(2), 215-225.

4 V. H. Mulimani and G. Rudrappa, Plant Foods Hum. Nutr., 1994, 46, 133-137.

5 F. Dou, M. Xi, J. Wang, X. Tian, L. Hong, H. Tang and A. Wen, Pharmazie, 2013, 68, 300-304.

6 B. Nickavar and N. Yousefian, J. Consum. Prot. Food Saf., 2011, 6, 191-195.

7 M. P. Mokoena, Molecules, 2017, 22(1255), 2-13.

8 A. Rzepkowska, Curr. Microbiol., 2015, 70, 183-194.

9 S. Surono and A. Hosono, Fermented Milks, 2011, 1, 477-482.

10 Y. Song, N. Shin and S. Baik, Food Sci. Biotechnol., 2014, 23(1), 1-8.

11 M. H. Helland, T. Wicklund and J. A. Narvhus, Food Res. Int., 2002, 35, 315-321.

12 Q. Zhang, Y. Wu and X. Fei, Medicina, 2016, 52(1), 28-34.

13 R. P. Srivastava, Indian J. Agric. Biochem., 2012, 25(1), 44-47. 14 I. T. Johnsona, J. Sci. Food Agric., 1993, 63, 201-209.

15 S. A. Valencia-Chamorro, Quinoa, Escuela Politécnica Nacional, 2003, pp. 4895-4902.

16 A. L. Lee, Y. P. Yu, J. F. Hsieh, M. I. Kuo, Y. S. Ma and C. P. Lu, Int. J. Biol. Macromol., 2018, 113, 601-606.

17 T. Yao and T. Rong, Mol. Nutr. Food Res., 2014, 15, 1930719318.

18 E. Apostolidis, Y. Kwon, R. Ghaedian and K. Shetty, Food Biotechnol., 2015, 19(2), 37-41.

19 N. Thanh, T. Nhien, N. T. Huy, D. T. Uyen, E. Deharo, P. Thi and L. Hoa, Trop. Med. Health, 2011, 39(4), 119-126.

20 K. B. Ishnava and D. M. Motisariya, International Journal of Pharmacognosy and Chinese Medicine, 2018, 2(3), 1-11.

21 M. I. Kazeem, J. V. Ogunbiyi and A. O. T. Ashafa, Trop. J. Pharm. Res., 2013, 12, 719-725.

22 M. Marrelli, F. Conforti, F. Araniti and G. A. Statti, Molecules, 2016, 27(1404), 1-20. 